\title{
El efecto de la responsabilidad organizacional sobre las actitudes hacia la contratación de personas con discapacidad*
}

\author{
The Effect of Organizational Responsibility in Attitudes \\ toward Hiring Individuals with Disadvantages
}

Recibido: julio 17 de 2013 | Revisado: mayo 17 de 2014 | Aceptado: junio 16 de 2014

\author{
BEATRIZ GANDARILlAS GUTIÉRREZ** \\ IE University, Madrid, España \\ BLANCA REQUERO *** \\ PABLO BRIÑOL \\ BEATRIZ ROJO**** \\ Universidad Autónoma de Madrid, España
}

doi.org/10.11144/Javeriana.UPSY13-4.eroa

Para citar este artículo: Gandarillas, B., Requero, B., Briñol, P., \& Rojo, B. (2014). El efecto de la responsabilidad organizacional sobre las actitudes hacia la contratación de personas con discapacidad. Universitas Psychologica, 13(4), 1615-1624. http:// dx.doi.org/10.11144/Javeriana.UPSY13-4.eroa

Este trabajo fue posible gracias al Group of Attitudes and Persuasion Spain (GAPS).

***Correo electrónico: beatriz.gandarillas@ie.edu

**** Correos electrónicos: blanca.requero@inv.uam.es, pablo.brinnol@uam.es

***** Correo electrónico: beatriz-rojo@hotmail.es

\section{RESUMEN}

La presente investigación estudia el cambio de actitudes en el contexto laboral hacia la contratación de personas con discapacidad. Fueron seleccionados aleatoriamente 80 trabajadores de distintas empresas para participar de forma voluntaria y anónima en esta investigación. Se examinó el efecto de la responsabilidad organizacional de los participantes sobre el procesamiento de mensajes persuasivos y las actitudes hacia la incorporación de trabajadores de este colectivo minoritario. Mediante un análisis de varianza, se encontró que los participantes que informaron tener (vs. no tener) responsabilidad sobre otras personas en sus empresas discriminaron en mayor medida entre los argumentos fuertes y débiles de la propuesta persuasiva. En línea con la investigación previa sobre responsabilidad personal y persuasión, estos resultados sugieren que la responsabilidad organizacional influye sobre la cantidad de procesamiento o sobre la validez de los pensamientos generados en respuesta a la propuesta.

Palabras clave

Actitudes; persuasión; cognición social; metacognición

\section{A B S T R A C T}

The present research studies how to promote attitudes toward hiring more people with disabilities in the job market. Eighty workers of different companies were selected randomly to take part voluntarily and anonymously in this study. We examined the effect of organizational responsibility on the processing of persuasive messages and attitudes toward incorporating more workers pertaining to this minority group. By means of an analysis of variance, we found that the participants who reported to have (vs. not to have) responsibility over other employees in their organizations were more likely to discriminate between the strong and weak arguments of the persuasive proposal. In line with previous research on personal responsibility and persuasion, these results suggest that organizational responsibility can influence the amount of processing or the perceived validity of the thoughts generated in response to the proposal.

Keywords

Attitudes; persuasion; social cognition; metacognition 


\section{Introducción}

Las personas con discapacidad son un colectivo históricamente excluido del mercado de trabajo y con especiales dificultades de inserción laboral. Según el Instituto Nacional de Estadística, la participación laboral de personas con discapacidad es un $40 \%$ menor que las personas que no pertenecen a este colectivo (Instituto Nacional de Estadística [INE], 2011). Además de la exclusión laboral, las personas con discapacidad que trabajan, con frecuencia son estigmatizadas, siendo objeto de numerosos estereotipos sociales y víctimas de discriminación (Muñoz, Pérez, Crespo, \& Guillén, 2009). Sería deseable contribuir a la mejora de la situación de este colectivo, reduciendo el estigma y generando actitudes más favorables hacia su inserción laboral. Precisamente, el objetivo del presente trabajo consiste en examinar el cambio de actitudes hacia este colectivo minoritario.

La investigación contemporánea sobre cambio de actitudes ha demostrado que la persuasión puede ocurrir tanto cuando las personas piensan detenidamente sobre la información de una propuesta persuasiva como cuando piensan poco en la propuesta (Petty \& Briñol, 2012). Esta distinción entre alta y baja elaboración cognitiva, o entre procesamiento delibrado y automático, es fundamental para entender la eficacia de los procesos implicados en la persuasión (para una revisión sobre modelos duales, véanse Sherman, Gawronski, \& Trope, 2014).

En el contexto de la persuasión, destaca el Modelo de Probabilidad de Elaboración ([ELM]; Petty $\&$ Briñol, 2012; Petty \& Cacioppo, 1986) que postula la existencia de un continuo de elaboración cuyos extremos constituyen dos vías o rutas, mediante las cuales los individuos procesan la información persuasiva que reciben. La primera recibe el nombre de ruta central y se refiere a procesos psicológicos de alta elaboración mental, mientras que la segunda es denominada ruta periférica y hace referencia a procesos que requieren una menor elaboración. A través de la ruta central, las personas utilizan su experiencia y conocimientos previos para analizar cuidadosamente toda la información que consideran relevante sobre el objeto de actitud. En cambio, a través de la ruta periférica, las personas utilizan procesos relativamente simples, confiando en claves sencillas y pistas heurísticas relacionadas con la propuesta (Petty \& Briñol, 2010; Petty \& Wegener, 1998; para una revisión histórica, véanse, Briñol \& Petty, 2012).

A lo largo de los años, se han identificado diversas variables que pueden afectar la probabilidad de elaboración y, por tanto, la medida en la que las personas siguen una u otra ruta hacia la persuasión. Estas variables implican tanto aspectos del individuo como de la situación y tienen que ver tanto con la capacidad como con la motivación para procesar la información persuasiva (para una revisión sobre estas variables, véanse, Briñol, De la Corte, \& Becerra, 2001).

De entre todas las variables que afectan la motivación para pensar sobre una propuesta, probablemente la relevancia personal o importancia del tema ha sido una de las que más atención ha recibido (Johnson \& Eagly, 1989; Leippe \& Elkin, 1987). La idea central del concepto de relevancia personal es que cuando las personas piensan que un mensaje versa sobre un tema de gran importancia para ellos o está asociado con sus intereses personales, se incrementa su motivación para pensar sobre la información contenida en dicho mensaje. Como resultado de un mayor análisis de la información, la relevancia personal suele producir un aumento del efecto de la calidad de los argumentos contenidos en el mensaje (Petty \& Cacioppo, 1990). Sin duda, una de las técnicas más eficaces para discriminar cuánto piensa una persona (y por tanto, para examinar el tipo de ruta, periférica o central, hacia la persuasión) consiste en variar la calidad de los argumentos de una misma propuesta persuasiva (Petty, Wells, \& Brock, 1976). En concreto, esta técnica consiste en comparar el impacto persuasivo de un mensaje compuesto por argumentos fuertes con otro compuesto, ambos por argumentos débiles a favor de una misma propuesta. De este modo, cuanto mayor es la diferencia de cambio de actitud producido por un mensaje fuerte y otro débil mayor es la cantidad de elaboración que se puede inferir (Briñol, Horcajo, Becerra, Valle, \& Gallardo, 2004; Briñol, Petty, Valle, Rucker, \& Becerra, 2007; Maio, Bell, \& Esses, 1996). 
En el estudio clásico de Petty y Cacioppo (1979) sobre relevancia personal y persuasión, se pidió a los participantes que evaluaran una propuesta que pretendía introducir exámenes finales obligatorios para la graduación. Para manipular la relevancia del tema, esta medida se planteaba llevar a cabo en ese mismo curso (alta relevancia, ya que les afectaba directamente a ellos) o dentro de diez años (baja relevancia). A continuación, todos los participantes recibían un mensaje sobre la propuesta que contenía bien argumentos fuertemente convincentes o bien argumentos débiles y sin méritos. Como cabía esperar, los participantes a quienes se les indujo alta relevancia sobre el tema mostraron un mayor efecto de la calidad de los argumentos. Es decir, comparado con los participantes de baja relevancia, aquellos a los que les iba a afectar la propuesta mostraron mayor persuasión para el mensaje fuerte y menor persuasión para el débil.

La relevancia personal puede variar en función de otros factores además de si una propuesta afecta directamente a la persona o no, como se ha descrito en la investigación anterior. Por ejemplo, la relevancia personal suele aumentar para cualquier aspecto relacionado con uno mismo (Blankenship $\&$ Wegener, 2008), incluyendo tanto su identidad grupal (Petty, Wheeler, \& Bizer, 2000) como sus valores y personalidad (Petty \& Cacioppo, 1990).

Entre estos factores de relevancia personal, cabe destacar por su importancia para la presente investigación el que tiene que ver con la responsabilidad. Petty, Harkins y Williams (1980) llevaron a cabo un estudio donde pedían a los participantes evaluar un artículo sobre la idea de incluir un examen final para poder graduarse. Todos los participantes leían una versión fuerte o una débil de esta propuesta. Además, se dijo a los participantes que ellos eran los únicos que iban a evaluar la propuesta (alta responsabilidad) o que formaban parte de un colectivo numeroso de evaluación (difusión de responsabilidad). Como se esperaba, la responsabilidad aumentó el efecto de la calidad de los argumentos, con mayor persuasión para el mensaje fuerte y menor para el débil.

La responsabilidad personal puede variar en función de otras variables más allá del número de personas encargadas de evaluar una propuesta. La presente investigación examina en qué medida la responsabilidad sobre otras personas puede funcionar como una variable de relevancia personal. En el caso de que la responsabilidad sobre otras personas funcione como otras formas de responsabilidad general y, por tanto, aumente el procesamiento, entonces, se esperarían resultados similares a los encontrados anteriormente. Por el contrario, en el caso de que la responsabilidad personal actúe de forma diferente a otras formas de responsabilidad, sus efectos sobre la persuasión podrían ser distintos.

Un ejemplo de ello podría ser la asociación entre la responsabilidad y el poder, ya que el poder induce a un estado de confianza en los propios puntos de vista que reduce la motivación para procesar (Fiske, 1993). En una investigación llevada a cabo por Briñol, Petty, Valle, Becerra y Rucker (2007, Experimento 2), se pidió a los participantes que desempeñaran el rol de jefe (alto poder) o subordinado (bajo poder) en una reunión de trabajo. A continuación, todos los participantes leyeron una propuesta persuasiva compuesta por argumentos fuertes o débiles a favor de un nuevo teléfono móvil y se evaluaron sus actitudes hacia el producto. Los resultados mostraron que las personas asignadas al rol de poder procesaron menos (menor efecto persuasivo de la calidad de los argumentos) que aquellos asignados a la condición sin poder (para otro ejemplo similar, véanse, Johnson \& Lammers, 2012; See, Morrison, Rothman, \& Soll, 2011). Por tanto, si la responsabilidad sobre otras personas se asocia con poder, se podría esperar que en el presente estudio redujese el efecto de la calidad de los argumentos. Por último, en la medida en que la responsabilidad active tanto relevancia como confianza, y dado que estas dos asociaciones hacen predicciones opuestas para el procesamiento, es posible que en el presente estudio no se observen resultados con respecto a la calidad de los argumentos.

En resumen, la responsabilidad sobre otras personas puede aumentar la responsabilidad en general y con ello la motivación para procesar y elaborar la información de los mensajes. De esta manera, se espera que quienes tienen responsabilidad serán capaces de discriminar entre mensajes fuertes y dé- 
biles en mayor medida que quienes no la tienen. Por el contrario, la responsabilidad sobre las personas también podría llevar a un aumento de la sensación de poder y con ello al incremento de la confianza, reduciendo así el procesamiento de la información y también el impacto de la calidad de los argumentos. Por último, otra posibilidad es que la responsabilidad sobre otras personas active ambas tendencias simultáneamente, cancelándose los efectos sobre el procesamiento. El objetivo principal del presente trabajo consiste en poner a prueba estas tres predicciones sobre la responsabilidad organizacional en el procesamiento de mensajes persuasivos.

\section{Método}

\section{Participantes}

Ochenta trabajadores participaron anónima y voluntariamente en el estudio (29 hombres y 51 mujeres, con un rango de edad entre los 21 y 63 años; $M=$ 42.01; $D E=12.7)$. El género y la edad no influyeron sobre los resultados. Es decir, no se encontraron efectos significativos de estas variables demográficas. Los participantes pertenecían a distintas empresas de diferentes sectores que fueron seleccionadas por criterios de proximidad y conveniencia ${ }^{1}$. Los resultados tampoco variaron en función del tipo de empresa ni de actividad de negocio.

\section{Diseño}

Diseño cuasiexperimental constituido por una variable independiente manipulada experimentalmente (calidad de los argumentos: fuerte vs. débil) y una segunda variable independiente medida posteriormente a la manipulación experimental y de naturaleza dicotómica (responsabilidad organiza-

1 Las empresas participantes pertenecían a los siguiente sectores: Comercio y hostelería (Bershka, Canon, Danone, Nivea, Daniel S.L, Clínica Baviera), Transportes, almacenamiento y comunicaciones (Aena, Airbuss, Metro de Madrid), Instituciones financieras, seguros y servicios a empresas (KPMG, Limpiezas Rodríguez), Servicios comunitarios, sociales y personales (Fundación Juan XXIII e Ibergrupo, Ministerio de Economía y Hacienda, Departamento Filosofía Universidad Autónoma de Madrid). cional: con responsabilidad vs. sin responsabilidad). La influencia de estas dos variables independientes se evaluó sobre las actitudes hacia la propuesta persuasiva que constituyó la variable dependiente fundamental del estudio.

\section{Procedimiento}

La investigación se presentó como un estudio de la Universidad Autónoma de Madrid (UAM), para fomentar la contratación en el ámbito organizacional de personas con discapacidad. Concretamente, a los participantes se les dijo que iban a formar parte en un estudio que consistía en evaluar la propuesta de la inserción laboral de personas con discapacidad física y/o mental y que había sido avalado por la UAM. A todos los participantes se les entregó un cuestionario que contenía las tareas que debían realizar y las instrucciones necesarias para llevarlas a cabo. En primer lugar, los participantes leyeron un mensaje que contenía argumentos a favor de la propuesta. La mitad de los participantes recibió un mensaje compuesto por argumentos fuertes y la otra mitad recibió un mensaje compuesto por argumentos débiles. Esta diferencia constituye la manipulación de la variable independiente, calidad de los argumentos. Después de leer el mensaje, se pidió a todos los participantes que informaran de sus actitudes hacia la contratación de personas con discapacidad. Tras completar la medida de actitudes, los participantes contestaron a una serie de preguntas adicionales relacionadas tanto con la tarea (e. g., esfuerzo, confianza) como con su cargo en la empresa en la que trabajaban. Específicamente, entre las preguntas sobre su actividad empresarial se preguntó a los participantes si tenían o no otras personas a su cargo. En función de las respuestas a esta pregunta, los participantes fueron clasificados en dos grupos, uno con responsabilidad sobre otras personas y otro sin responsabilidad. Al final de la sesión, se informó a los a los participantes de cuáles fueron los objetivos principales de la investigación, ofreciéndose además la posibilidad de ampliar la información sobre la misma. Por otro lado, se agradeció su participación y se solicitó permiso para el análisis de sus respuestas, garantizándoles la 
confidencialidad y anonimato. Por tanto, el estudio contó con el consentimiento informado por parte de los participantes.

\section{Variables independientes}

\section{Calidad de los argumentos}

Los participantes fueron asignados aleatoriamente a una de las dos condiciones de esta variable independiente. Específicamente, los participantes recibieron un mensaje para fomentar la contratación de personas con discapacidad que contenía argumentos fuertes o débiles. El mensaje fuerte incluía 5 argumentos muy convincentes y el mensaje débil estaba compuesto por 5 argumentos muy poco convincentes, todos ellos claramente a favor de la contratación de personas con discapacidad. Algunos ejemplos de los argumentos del mensaje fuerte fueron: "Mejora el clima laboral ya que los trabajadores eliminan muchos estereotipos y su conducta se modifica positivamente hacia la tolerancia y apertura", "Se adaptan mejor a los cambios, son más innovadoras incidiendo en los beneficios de la empresa, por la escucha activa y la diversidad del personal", "Oportunidad de favorecer la responsabilidad social corporativa, así como el bienestar psicológico de las empresas" y "Bonificaciones fiscales hasta el 100\% de la cuota de la seguridad social por contratar trabajadores con certificado de discapacidad".

Ejemplos de argumentos débiles fueron: "Mejora el clima laboral ya que tienden a dejar de lado cotilleos que puedan ocasionar deficiencias en el entorno laboral", "Suelen ser más puntuales que los demás", "Favorece la responsabilidad social corporativa porque es una política organizacional que está de moda. Es una cuestión que aparece en muchos foros y estudios en la actualidad", y "Son personas que fuman menos y por tanto no realizan tantos descansos para salir a fumar cigarrillos". La eficacia de comparar el impacto persuasivo relativo de mensajes fuertes y débiles se ha puesto de manifiesto en varias investigaciones previas (para un ejemplo en castellano, véanse, Briñol et al., 2004).

\section{Responsabilidad organizacional}

Se preguntó a los participantes si tenían o no responsabilidad sobre otras personas. En concreto, se utilizó la siguiente pregunta "iTiene personal a su cargo?”. Sólo se ofrecía la posibilidad de contestar de forma dicotómica (sí o no) a esta cuestión. Por tanto, los participantes fueron clasificados en dos grupos en función de sus respuestas, uno con responsabilidad sobre otras personas y otro sin ella.

\section{Variables dependientes}

\section{Actitudes}

Las actitudes hacia la contratación de personas con discapacidad fueron evaluadas en una escala compuesta por 4 ítems tipo Likert de 9 puntos, siendo 1 nada y 9 totalmente, que incluía los adjetivos interesante, útil, beneficiosa y positiva. Una vez recodificado el valor de los ítems redactados de forma inversa, se creó un índice compuesto por los cuatro ítems $(\alpha=0.67)$.

\section{Esfuerzo cognitivo}

Para evaluar si los participantes informaban haber pensado sobre la información presentada en el mensaje persuasivo, se utilizó una escala tipo Likert de 9 puntos, siendo 1 no pensé y 9 pensé mucho.

\section{Confianza en los pensamientos}

Para evaluar la confianza en los pensamientos que generaron los participantes se utilizó una escala compuesta por 1 ítem tipo Likert de 9 puntos, siendo 1 ninguna confianza y 9 mucha confianza.

\section{Análisis de datos}

Las medidas dependientes fueron sometidas a un análisis de varianza (ANOVA) de dos factores (calidad de los argumentos y responsabilidad organizacional) a través del paquete estadístico SPSS (versión 20.0 para Windows). Para evaluar la bondad de ajuste de la variable dependiente de actitudes, 
se utilizó el programa Mplus (versión 6; Muthén \& Muthén, 2006-2010). Por último, mediante un procedimiento de estimación de máxima verosimilitud robusta (MLR), se obtuvieron la raíz del error cuadrático medio de aproximación (RMSEA), el índice de ajuste comparativo (CFI), el índice raíz cuadrática media de residuales (SRMR) y la razón de chi cuadrado sobre los grados de libertad $\left(\chi^{2} / g l\right)$.

\section{Resultados}

\section{Actitudes hacia la propuesta}

Los indicadores de bondad de ajuste mostraron valores adecuados para la medida de las actitudes $\left(\mathrm{RMSEA}=0 ; \mathrm{CFI}=1 ; \mathrm{SRMR}=0.02 ; \chi^{2} / g l=\right.$ 6.44). Esta variable dependiente fue sometida a un análisis de varianza (ANOVA) 2 (calidad de los argumentos: fuertes vs. débiles) X 2 (responsabilidad organizacional: con responsabilidad vs. sin responsabilidad). El ANOVA sobre el índice de actitudes mostró un efecto principal de la variable calidad de los argumentos, $F(1,76)=12.36 ; p<0.001, \eta_{p}{ }^{2}$ $=0.14$. Este efecto indica que los participantes que recibieron el mensaje compuesto por argumentos fuertes mostraron actitudes significativamente más favorables hacia la propuesta $(M=7.23 ; D E=1.31)$ que los participantes que recibieron el mensaje compuesto por argumentos débiles $(\mathrm{M}=6.92 ; \mathrm{DE}=$ 1.5). No se encontró efecto principal de la variable responsabilidad organizacional, $F(1,76)=0.73 ; p$ $=0.4, \eta_{\mathrm{p}}^{2}=0.01$.

Tal y como ilustra la Figura 1, este efecto principal fue cualificado por la interacción entre la calidad de los argumentos y responsabilidad organizacional, $F_{(1,76)}=10.88 ; p=0.001, \eta_{p}^{2}=0.125$. Esta interacción muestra que, en la condición con responsabilidad organizacional, la diferencia entre el efecto persuasivo del mensaje fuerte $(M=8.01$; $D E=0.91)$ y el mensaje débil $(M=5.82 ; D E=$ 2.24) resultó estadísticamente significativa, $F_{(1,76)}=$ 15.68; $p<0.001, \eta_{\mathrm{p}}{ }^{2}=0.171$. Sin embargo, para el grupo sin responsabilidad organizacional, no hubo diferencias en las actitudes entre quienes recibieron el mensaje compuesto por argumentos fuertes ( $M$ $=7.22 ; \mathrm{DE}=1.01)$ y quienes recibieron el mensaje compuesto por argumentos débiles $(\mathrm{M}=7.15 ; \mathrm{DE}$ $=1.21), F_{(1,76)}=0.04 ; p=0.83, \eta_{p}^{2}=0.001$. Es decir, la responsabilidad de tener personas a su cargo aumentó el efecto de la calidad de los argumentos.

Descrita de otra forma, esta interacción demuestra que entre los participantes que recibieron los

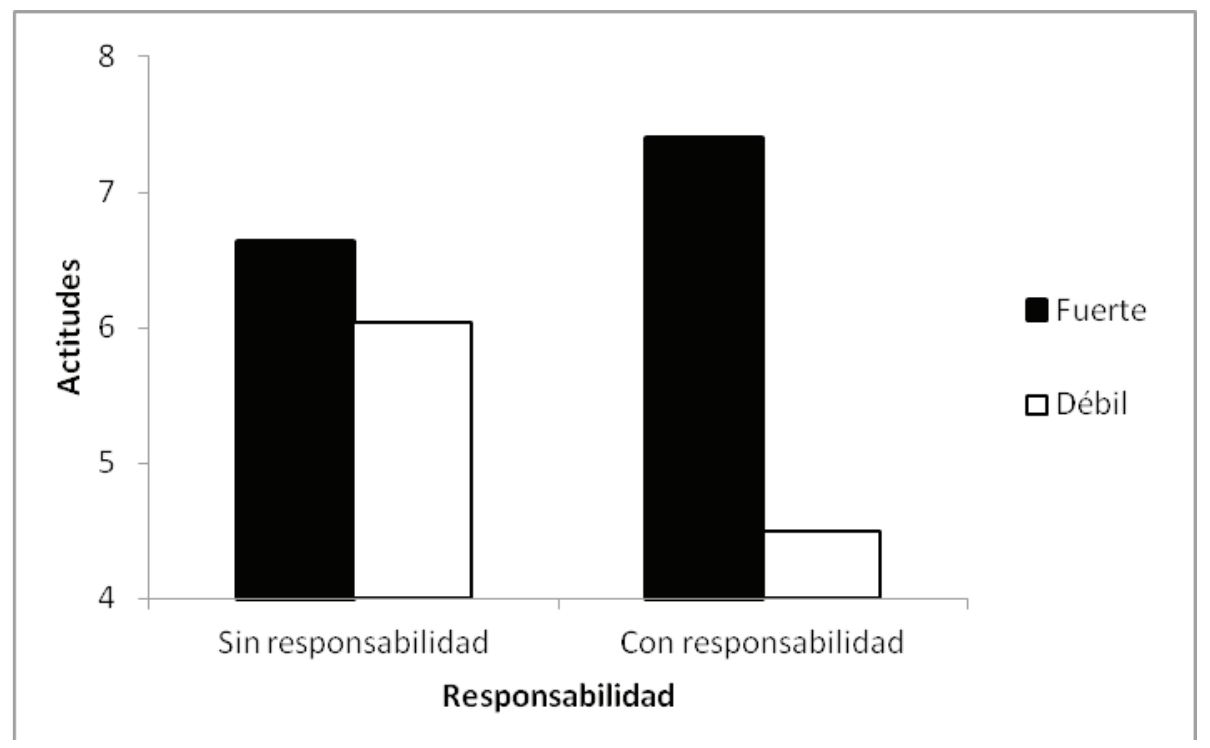

Figura 1. Actitudes hacia la contratación de personas con discapacidad en función de la responsabilidad organizacional y la calidad de los argumentos

Fuente: elaboración propia 
argumentos débiles, aquellos sin responsabilidad mostraron actitudes más favorables hacia la propuesta $(M=7.15 ; \mathrm{DE}=1.21)$ que los que tenían responsabilidad $(\mathrm{M}=5.82 ; \mathrm{DE}=2.24), \mathrm{F}_{(1,76)}=$ 6.91; $p=0.01, \eta_{p}^{2}=0.083$. Por el contrario, los participantes que recibieron argumentos fuertes, aquellos sin responsabilidad $(\mathrm{M}=7.22 ; \mathrm{DE}=1.01)$ mostraron actitudes menos favorables que aquellos con responsabilidad $(\mathrm{M}=8.01 ; \mathrm{DE}=0.91), \mathrm{F}_{(1,76)}$ $=2.73 ; p=0.05, \eta_{\mathrm{p}}^{2}=0.05$.

\section{Esfuerzo cognitivo}

El ANOVA 2 × 2 reveló un efecto principal significativo de la variable independiente, responsabilidad organizacional, sobre el esfuerzo cognitivo $F_{(1,71)}=5.32 ; p=0.02, \eta_{p}{ }^{2}=0.07$, indicando que los participantes informaron haber pensado más en la condición con responsabilidad $(\mathrm{M}=8.18 ; \mathrm{DE}=$ 0.79) que en la condición sin responsabilidad (M $=7.43 ; \mathrm{DE}=1.33)$. No se encontraron resultados significativos ni del efecto principal de la variable calidad de los argumentos $F_{(1,71)}=0.06 ; p>0.79$, $\eta_{\mathrm{p}}{ }^{2}=0.001$, ni de la interacción $F_{(1,71)}=0.19 ; p=$ $0.66, \eta_{p}^{2}=0.003$.

\section{Confianza en los pensamientos}

El ANOVA 2 X 2 no reveló ningún efecto significativo sobre esta medida, ni de la calidad de los argumentos, $F_{(1,72)}=1.13 ; p=0.29, \eta_{p}^{2}=0.015$, ni de la responsabilidad organizacional, $F_{(1,72)}=1.45$; $p=0.23, \eta_{\mathrm{p}}{ }^{2}=0.02$, ni tampoco de la interacción entre las mismas, $F_{(1,72)}=0.005 ; p=0.95, \eta_{p}{ }^{2}=0$.

\section{Discusión}

La investigación previa sobre persuasión y relevancia personal demuestra que cuando a las personas se les presenta un mensaje sobre un tema de gran importancia para ellas, se incrementa su motivación por procesar dicho mensaje (Petty \& Cacioppo, 1990). En una investigación previa sobre este tema, la relevancia personal fue manipulada experimentalmente, a partir del número de personas que tenían que evaluar un mensaje (Petty et al., 1980). En el presente trabajo de investigación se ha estudiado la relevancia personal a través de la responsabilidad que informaron tener los trabajadores sobre otras personas. Se plantearon tres posibles hipótesis del efecto de esta variable sobre el procesamiento de mensajes persuasivos hacia personas con discapacidad: la responsabilidad sobre otras personas podía aumentar el procesamiento, disminuirlo o no tener impacto sobre el mismo.

De estas tres hipótesis, los resultados muestran que la responsabilidad llevó a un mayor procesamiento, indicado por un mayor impacto de la calidad de los argumentos. Es decir, los participantes con personas a su cargo mostraron actitudes más favorables hacia la contratación de personas con discapacidad, cuando recibieron argumentos fuertes que cuando recibieron argumentos débiles. Por el contrario, las personas que no tienen responsabilidad organizacional, no mostraron diferencias en sus actitudes cuando se les presentó un mensaje fuerte o uno débil.

Estos resultados son compatibles con la idea de que la responsabilidad sobre otras personas puede influir en el cambio de actitudes, de la misma manera que otras formas de responsabilidad. A pesar de que el patrón de cambio de actitudes en función de esta nueva forma de operativizar las variables es claro, todavía quedan algunos aspectos pendientes de esclarecer. En primer lugar, no está claro si el efecto sobre las actitudes se debe a un proceso cognitivo de elaboración o más bien a un proceso metacognitivo de validación. Podría ser que el mayor efecto de la calidad de los argumentos sobre las actitudes se debiese a una mayor confianza en los pensamientos por parte de aquellos con personas a su cargo (Briñol et al., 2007; Briñol, Petty, \& Stavraki, 2012; Briñol, Valle, \& Becerra, 2008, para una revisión; DeMarree, Briñol, \& Petty, en prensa). Aunque la investigación previa sobre poder deja clara esta posibilidad, los resultados del presente estudio no muestran efectos significativos sobre la confianza. Además, trabajos anteriores han establecido claramente que los mecanismos de validación operan con mayor probabilidad cuando las variables (como la relevancia) se inducen después (en lugar de antes) de procesar el mensaje (Briñol \& Petty, 
2009). Por tanto, futuros trabajos deberían manipular experimentalmente el orden de presentación de las variables independientes.

Además del efecto del poder sobre el procesamiento y la validación de mensajes persuasivos, trabajos previos han examinado el papel de otras variables en el contexto del cambio de actitudes hacia propuestas organizacionales. Por ejemplo, DeMiguel et al. (2009) encontraron que las personas con mayor estrés organizacional discriminaron menos entre argumentos fuertes y débiles, al procesar una propuesta de cambio organizacional. Aplicando esos resultados al presente paradigma, se podría especular sobre la posibilidad de que las personas con menor responsabilidad organizacional tuvieran un mayor estrés. Aunque esto pueda parecer poco probable desde un punto de vista de los modelos situacionales del estrés (según los cuales a mayor responsabilidad mayor probabilidad de padecer estrés), sería compatible con otros enfoques organizacionales, según los cuales las personas pueden tener estrés como resultado precisamente de no tener suficiente responsabilidad, autonomía o cualificación para desempeñar su trabajo (García, Silla, Peiró, \& Fortes-Ferreira, 2006). Por otro lado, se podría mencionar también la relación entre responsabilidad y estados de ánimo. La investigación previa demuestra que las emociones negativas están asociadas con una alta discriminación entre mensajes persuasivos fuertes y débiles (Briñol, Gandarillas, Horcajo, \& Becerra, 2010). Por tanto, existe la posibilidad de que las personas con responsabilidad sobre otras experimenten peores estados de ánimo y, como consecuencia, procesen más los mensajes persuasivos. Tomados conjuntamente ambos planteamientos, parece claro que futuros trabajos deberán incluir medidas tanto de estrés como de estados de ánimo y satisfacción laboral (López-Araujo, Osca, \& Peiró, 2007). Por último, trabajos previos han mostrado que la responsabilidad puede estar asociada con un aumento de la vigilancia, lo cual podría estar asociado con un mayor procesamiento incluso en personas con puestos de poder (Magee, 2009; Min \& Kim, 2013).

Además de incluir un mayor número de medidas, una limitación reseñable de la presente investigación tendría que ver con la ausencia de medidas objetivas sobre la fuerza de las actitudes, tales como la resistencia y la estabilidad de los cambios obtenidos (Petty \& Krosnick, 1995). La inclusión de estos indicadores en futuros estudios podría ayudar a aclarar en qué medida la elaboración de los pensamientos es el proceso responsable de tales resultados.

Por otra parte, en este estudio la variable fundamental, responsabilidad sobre otras personas, no fue manipulada experimentalmente sino medida a través del autoinforme de los participantes. Aunque esto puede aumentar la validez ecológica del estudio, de cara a otros posibles trabajos sería deseable establecer un diseño experimental en el que los participantes fueran asignados aleatoriamente a condiciones con mayor o menor responsabilidad sobre otras personas. Entre otras cosas, eso daría mayores garantías a la hora de establecer relaciones causales y permitiría equiparar de forma precisa la distribución de participantes en las condiciones experimentales.

También relacionado con la medida de la responsabilidad, conviene señalar que en el presente estudio se utilizaron las respuestas proporcionadas por los participantes y, por tanto, la variable estudiada tiene que ver con la percepción subjetiva de la responsabilidad, que con frecuencia coincide con las responsabilidades objetivas sobre otras personas en términos de autoridad formal y distribución jerárquica del poder en las organizaciones. Sin embargo, la percepción subjetiva y la realidad objetiva constituyen dos constructos diferentes y puede haber discrepancias entre los mismos (e. g., personas con alta responsabilidad real que creen no tener ninguna responsabilidad y personas sin ninguna responsabilidad objetiva que creen tenerla). Por tanto, futuros trabajos deberían incluir no solo medidas de la percepción de la responsabilidad, sino también indicadores objetivos de esta variable. Por supuesto, otras medidas adicionales relacionadas con la responsabilidad sobre otras personas, como la motivación hacia el poder, también podrían ser de interés para futuros trabajos (e. g., Chen, Langner, $\&$ Mendoza-Denton, 2009).

Otro aspecto pendiente tiene que ver con la medida en que la responsabilidad sobre otras personas 
influye en el procesamiento de mensajes exclusivamente sobre la contratación de personas (como en este estudio) o sobre el procesamiento general de cualquier propuesta persuasiva aunque no tenga que ver con otras personas. Asímismo, convendría examinar en qué medida se pueden generalizar los resultados encontrados en este trabajo al cambio de actitudes hacia otros colectivos discriminados en el mundo laboral, más allá de las personas con discapacidad.

Por último, conviene señalar que los resultados de la presente investigación podrían tener aplicaciones prácticas para los procesos de selección. Por ejemplo, se podría dar mayor responsabilidad o, al menos, aumentar la creencia subjetiva de tener responsabilidad, a las personas encargadas de evaluar los currículos de posibles candidatos justo antes de evaluarlos. Según los resultados encontrados, eso contribuiría a la generación de actitudes favorables hacia las personas con discapacidad fuertemente cualificadas, facilitando su posterior inserción laboral. Como se ha puesto de manifiesto, esta práctica también limitaría la contratación potencial de candidatos poco cualificados.

\section{Referencias}

Blankenship, K. L., \& Wegener, D. T. (2008). Opening the mind to close it: Considering a message in light of important values increases message processing and later resistance to change. Journal of Personality and Social Psychology, 94(2), 196-213.

Briñol, P., De la Corte, L., \& Becerra, A. (2001). Quées persuasión. Madrid: Biblioteca Nueva.

Briñol, P., Gandarillas, B., Horcajo, J., \& Becerra, A. (2010). Emoción y metacognición: implicaciones para el cambio de actitud. Revista de Psicología Social, 25(2), 157-183.

Briñol, P., Horcajo, J., Becerra, A., Valle, C., \& Gallardo, I. (2004). El efecto de la ambivalencia evaluativa sobre el cambio de actitudes. Psicothema, 16(3), 373-377.

Briñol, P., \& Petty, R. E. (2009). Persuasion: Insights from the self-validation hypothesis. En M. P. Zanna (Ed.), Advances in experimental social psychology (Vol. 41, pp. 69-118). New York: Academic Press.
Briñol, P., \& Petty, R. E. (2012). The history of attitudes and persuasion research. En A. Kruglanski \& W. Stroebe (Eds.), Handbook of the history of social psychology (pp. 285-320). New York: Psychology Press.

Briñol, P., Petty, R. E., \& Stavraki, M. (2012). Power increases the reliance on first-impression thoughts. Revista de Psicología Social, 27(3), 293-303.

Briñol, P., Petty, R. E., Valle, C., Rucker, D. D., \& Becerra, A. (2007). The effects of message recipients' power before and after persuasión: A selfvalidation analysis. Journal of Personality and Social Psychology, 93(6), 1040-1053.

Briñol, P., Valle, C., \& Becerra, A. (2008). El efecto del poder sobre la confianza en los propios pensamientos. En J. F. Morales, C. Huici, E. Gaviria \& A. Gómez (Eds.), Método, teoría e investigación en psicología social (pp. 662-685). Madrid: Pearson Prentice Hall.

Chen, S., Langner, C. A., \& Mendoza-Denton, R. (2009). When dispositional and role power fit: Implications for self-expression and self-other congruence. Journal of Personality and Social Psychology, 96(3), 710-727.

DeMarree, K. G., Briñol, P., \& Petty, R. E. (2014). The effects of power on prosocial outcomes: A self-validation analysis. Journal of Economical Psychology.

De Miguel, J., Gallardo, I., Horcajo, J., Becerra, A., Aguilar, P., \& Briñol, P. (2009). El efecto del estrés sobre el procesamiento de mensajes persuasivos. Revista de Psicología Social, 24(3), 399-409.

España, Instituto Nacional de Estadística. (2011). El empleo de las personas con discapacidad. Explotación de la encuesta de población activa y de la base estatal de personas con discapacidad 2009-2010 (Informe anual). Madrid: Autor.

Fiske, S. T. (1993). Controlling other people: The impact of power in stereotyping. American Psychologist, 48(6), 621-628.

García, F. J., Silla, I., Peiró, J. M., \& Fortes-Ferreira, L. (2006). El estado del contrato psicológico y su relación con la salud psicológica de los empleados. Psicothema, 18(2), 256-262.

Johnson, B., \& Eagly, A. H. (1989). Effects of involvement on persuasion: A meta-analysis. Psychological Bulletin, 106(2), 290-314. 
Johnson, C. S., \& Lammers, J. (2012). The powerful disregard social comparison information. Journal of Experimental Social Psychology, 48(1), 329-334.

Leippe, M. R., \& Elkin, R. A. (1987). When motives clash: Issue involvement and response involvement as determinants of persuasion. Journal of Personality and Social Psychology, 52(2), 269-278.

López-Araujo, B., Osca, A., \& Peiró, J. M. (2007). El papel modulador de la implicación con el trabajo en la relación entre el estrés y la satisfacción laboral. Psicothema, 19(1), 81-87.

Magee, J. C. (2009). Seeing power in action: The roles of deliberation, implementation, and action in inferences of power. Journal of Experimental Social Psychology, 45(1), 1-14.

Maio, G. R., Bell, D. E., \& Esses, V. M. (1996). Ambivalence and persuasion: The processing of messages about immigrant groups. Journal of Experimental Social Psychology, 32(6), 513-536.

Min, D., \& Kim, J. -H. (2013). Is power powerful? Power, confidence, and goal pursuit. International Journal of Research in Marketing, 30(3), 265-275.

Muñoz, M., Pérez, E., Crespo, M., \& Guillén, A. (2009). Estigma y enfermedad mental: análisis del rechazo social que sufren las personas con enfermedad mental. Madrid: Universidad Complutense de Madrid, Editorial Complutense, S.A.

Petty, R. E., \& Briñol, P. (2010). Attitude change. En R. F. Baumeister \& E. J. Finkel (Eds.), Advanced social psychology: The state of the science (pp. 217259). Oxford: Oxford University Press.

Petty, R. E., \& Briñol, P. (2012). The Elaboration Likelihood Model. En P. A. M. Van Lange, A. Kruglanski \& E. T. Higgins (Eds.), Handbook of social psychology (pp. 285-320). New York: Guilford Press.

Petty, R. E., \& Cacioppo, J. T. (1979). Issue involvement can increase or decrease persuasion by enhancing message-relevant cognitive responses. Journal of Personality and Social Psychology, 37(10), 1915-1926.

Petty, R. E., \& Cacioppo, J. (1986). Communication and persuasion: Central and peripheral routes to attitude change. New York: Springer Verlag.

Petty, R. E., \& Cacioppo, J. T. (1990). Involvement and persuasion: Tradition versus integration. Psychological Bulletin, 107(3), 367-374.

Petty, R. E., Harkins, S. G., \& Williams, K. D. (1980). The effects of group diffusion of cognitive effort on attitudes: An information processing view. Journal of Personality and Social Psychology, 38(1), 81-92.

Petty, R. E., \& Krosnick, J. A. (Eds.). (1995). Attitude strength: Antecedents and consequences. Mahwah, NJ: Erlbaum.

Petty, R. E., \& Wegener, D. T. (1998). Attitude change: Multiple roles for persuasion variables. En D. Gilbert, S. Fiske \& G. Lindzey (Eds.), The handbook of social psychology (4.a ed., Vol. 1, pp. 323-390). New York: McGraw-Hill.

Petty, R. E., Wells, G. L., \& Brock, T. C. (1976). Distraction can enhance or reduce yielding to propaganda. Thought disruption versus effort justification. Journal of Personality and Social Psychology, 34(5), 874-884.

Petty, R. E., Wheeler, S. C., \& Bizer, G. (2000). Matching effects in persuasion: An elaboration likelihood analysis. En G. Maio \& J. Olson (Eds.), Why we evaluate: Functions of attitudes (pp. 133. 162). Mahwah, NJ: Erlbaum.

See, K. E., Morrison, E. W., Rothman, N. B., \& Soll, J. B. (2011). The detrimental effects of power on confidence, advise taking, and accuracy. Organizational Behavior and Human Decision Processes, 116(2), 272-285.

Sherman, J. W., Gawronsk, B., \& Trope, Y. (Eds.). (2014). Dual-process theories of the social mind. New York: Guilford Press. 